\title{
Metaphysics and the Limit of the Natural Sciences Explanatory Power
}

\author{
Aleksandr Kulieshov \\ Cherkasy State Technological University, Ukraine \\ Department of Philosophical and Political Sciences, PhD in Philosophy, Associate Professor
}

\begin{abstract}
The article concern is the problem of natural sciences - metaphysics relations. The criterion of metaphysical knowledge as the knowledge about fundamental objects is formulated. It is argued that natural sciences and mathematics do not meet this criterion. They cannot be metaphysics being engaged in the study of exclusively non-fundamental entities. The inference is made that only the speculative metaphysics (if such metaphysics is possible) has the means to solve metaphysical problems relying entirely on its own foundation.
\end{abstract}

Keywords: metaphysics; natural sciences; naturalistic metaphysics; speculative metaphysics; fundamental knowledge; non-fundamental knowledge.

\section{LCC Subject Category: BF1-990}

DOI: 10.22178/pos.19-9

\section{Introduction}

Any knowledge or understanding of reality is found within the horizon of a certain world picture. That is why metaphysical perspective is inevitable for any cognitive activity of human beings. Such perspective can have both rational and irrational representations. Rational representation is known under the name of metaphysics as a branch of theoretical knowledge. The two main sources of this knowledge are experience and speculative thinking (or apriori reasoning). The latter is also derived from experience but undergoes crucial break up with it by means of abstraction. Empirical knowledge and to great extent speculative one (exactly - mathematical) are represented within the sphere of theoretical cognition by natural and social sciences. The task of theoretical modelling the world as a whole is pursued now by natural sciences, first of all by physics. It may seem that in this situation there is no place left for metaphysics as a separate theoretical discipline. The first question concerning metaphysics therefore is: in what relation is it to natural (and social as continuous with them) sciences? The popularity of this question in nowadays philosophical papers confirms its priority for metaphysics.

One of the two variants is logically possible: either metaphysics coincides with natural sciences or not. In the first case metaphysics appears to be the part of natural sciences and not the reverse since natural sciences investigate not only the world as a whole but are going into details which are beyond the sphere of metaphysics. Such metaphysics within natural sciences deserves to be called naturalistic metaphysics. Defining the role played by naturalistic metaphysics in natural sciences one can discern two variants. Let us call them the weak and the strong versions of naturalistic metaphysics. The weak version acknowledges to some degree the autonomy granted to metaphysics within natural science but denies, figuratively speaking, the sovereignty of metaphysics. The strong version does not distinguish metaphysics from natural science (and even more radically - from physics). Physics (or natural science in general) from this point of view is itself metaphysics at the level of fundamental theories. Among the proponents of the strong version of naturalistic metaphysics there are plenty of physicists (perhaps, the majority of them). Suffice it to mention such reputable scholars as Steven Weinberg [13] and Stephen Hawking [8]. The weak version of naturalistic metaphysics is popular among philosophers (the most discussed in anglophone philosophical literature conception now is in the book of J. Ladyman and D. Ross [9]). Under naturalistic metaphysics the authors mean "a metaphysics that is motivated exclusively by attempts to unify hypotheses and theories that are taken seriously by contemporary science" $[9$, p. 1]. J. Wilson prefers to call it "the embedded conception" of metaphysics having in mind metaphysics embedded in other disciplines "with the directions of potential influence going both to and from metaphysics" [14, p. 94]. 
If we assume that metaphysics doesn't coincide with natural sciences, we have one or another version of speculative or a priori metaphysics. It is sometimes called analytical metaphysics, but this name does not seem good, because one can think that non-naturalistic metaphysics should be reduced to conceptual analysis in the spirit of P. Strawson's descriptive metaphysics which is not the case. (The understanding of metaphysics as organization and systematization of our concepts one can find in the paper of J. Benovsky [3]). Speculative metaphysics, at least in its foundation, is outside of natural sciences. Being exclusive knowledge about the world as a whole, while natural sciences are non-exclusive knowledge, speculative metaphysics can play the role of the basis of natural sciences. The two possible versions of speculative metaphysics, similarly to naturalistic metaphysics, are the strong and the weak one. The weak version suggests some overlap of the subject areas of metaphysics and natural science. In this version metaphysics adds a bit of its own specific explanations to common objects of knowledge with natural sciences. The conceptual apparatus of metaphysics partially includes the conceptual apparatus of physics. The strong version totally separates the subject areas of metaphysics and natural sciences. Metaphysics this way forms the knowledge about the special metaphysical - reality. There must be a correspondence, but not a coincidence among the objects studied by metaphysics and those studied by natural sciences.

The weak version of speculative metaphysics is widely spread among contemporary metaphysicians. In different forms it is expressed in many recent publications. J. Faye's leaves to metaphysics the region of hypotheses. "The distinction between science and metaphysics is a separation made, not by language, but by conditions of epistemic access: between what we can and cannot empirically know. The sciences come to an end at the point at which hypotheses are empirically underdetermined." [7, p. 65] Nonetheless philosophers cannot say something about the universe which physicists and cosmologists cannot either [7, p. 70]. K. Bennet agrees with the need to go some distance towards distinguishing metaphysics from science but "the line is unlikely to be crystal clear" [2, p.30]. As it is stated by M. Morganti, "metaphysics cannot be read off from science" but "naturalism about metaphysics should be understood as the view that metaphys- ics should preserve its autonomy but be studied in parallel with science, being put to the test of the empirical evidence..." [11, p. 69] M. Esfield's role for metaphysics is to enrich ontology produced by science at its foundations [6]. E. J. Lowe defines metaphysics as the formal study of all which is possible [10]. F. Andersen and A. J. R Becker advocate cooperation between natural sciences and metaphysics [1]. A. Chakravartty's claim is that "the metaphysical theses argued for by metaphysicians are not extracted from empirical content of science, as if they were there already simply waited to be mined. They are developed by means of a priori theorizing in the course of interpreting scientific claims" [5, p.25]. All the mentioned authors distinguish metaphysics and natural sciences to some extent and yet leave them in direct interaction.

The strong version of speculative metaphysics was typical for post-Kantian metaphysics of the XIX century (when the confrontation between philosophy and natural sciences became sufficiently acute). One of the most uncompromising formulations of that time belongs to F. Bradley, who claimed that "Nature by itself has no reality. It exists only as a form of appearance within the Absolute. In its isolation from that whole of feeling and experience it is an untrue abstraction" while "for physical science, the separation of one element from the whole is both justifiable and necessary" [4, p. 293]. Bradley's argumentation is based on the idea that the absolutely real is the absolute reality and nothing but it. Accordingly all the objects of scientific interest are more or less illusory. Nowadays science and philosophy quite reasonably reject this approach. However, I believe that concerning naturalisticmetaphysical controversy F. Bradley is rather right than wrong. The point is to find more powerful argumentation than outdated Bradley's.

The statement of L. Paul that metaphysics "describes features of the world that are more fundamental than those of natural science" looks promising in this sense [12, p. 5]. L. Paul bases his assumption on "the fact that many concepts of metaphysics are conceptually prior to the concepts of science" [12, p. 6]. It seems that there are no other arguments in his conception than those appealing to conceptual priority of the objects of metaphysics. He is apparently right in that "it is a mistake to think that one should first study science and then use it as the guide to one's metaphysical conclusions" [12, p. 6]. But this strong 
metaphysical claim requires more convincing argumentation. It is not entirely clear, whether the aforesaid conceptual priority has explicit ontological implications. In this context it looks both necessary and possible to strengthen the arguments in favour of the independence of metaphysics from natural sciences. The main and decisive question is not whether metaphysics is something other than natural sciences, but whether natural sciences are capable of being metaphysics, that is, to obtain metaphysical results.

The purpose of this article is to demonstrate that the answer on the last question is negative. It will first be shown that the argument from conceptual priority of metaphysical objects is not strong enough. After that the criterion of metaphysical knowledge will be formulated. Finally, it will be demonstrated why the findings of natural sciences do not meet this criterion.

\section{Result and Discussion}

It is necessary for our purposes to discern the objects of metaphysics and the objects of natural sciences (for simplicity we might call the latter "physical objects"). These objects are understood strictly as what is studied by metaphysics or natural sciences (physics) regardless of its mode of being. Physical and metaphysical objects can coincide in their existence completely or partially, but it is also not taken into account. It is obvious that the objects of metaphysics are more (at least, not less) general and abstract than physical objects. Nevertheless it does not give them ontological priority. It is not the place here to discuss philosophical controversy of the metaphysical nominalists and realists. But the physicists in search of the final theory may state that they have found some specific and individual entities which determine all the general features of the world. This claim needs to be justified. It can also be refuted (and should be refuted) but it cannot be done on the ground of conceptual priority. The problem of ontological priority is not solvable within the scope of conceptual priority. Meanwhile physics in this dispute have the advantage of being able to present results based on empirical data. What have been said does not mean that naturalistic metaphysics is a correct version of metaphysics. It means that the arguments based on conceptual priority are not sufficient to justify speculative metaphysics. The problem solution is not in proving the conceptual and ontological priority of the metaphysical objects. It is rather in defining the metaphysical objects as ontologically prior.

The knowledge of any object cannot be complete if we can't explain its existence, that is, to find the necessary and sufficient conditions under which it exists. Different conditions in practice are taken into account by researchers (causal, structural etc.), but for the discussion it is only important that the entire set of conditions can be qualified as the ground of a particular object existence. With respect to their grounds objects can be divided into two classes. At our disposal there are the fundamental objects that is, those which have their grounds in themselves. It is not crucial for our reasoning whether they exist in reality or not. The class of the fundamental objects includes first of all those which are self-grounded but we can also add here the objects which are directly grounded by the self-grounded objects or connected with them by the finite sequence of grounds. Let's call such objects fundamentaldependent. The class of the fundamental objects, thus, will be considered broadly, including both the self-grounded objects and the fundamentaldependent. If we make the objects of cognition out of the fundamental objects in a broad sense, the knowledge about them can also be called the fundamental knowledge. It's worth noting that the fundamental knowledge arises only when we consider the fundamental objects exactly as such. The same objects can be examined in a different context then the knowledge will not be fundamental.

The foregoing makes it clear that there are the non-fundamental objects and the nonfundamental knowledge as well. The objects are non-fundamental so far as they are taken in their grounding dependencies from other nonfundamental objects regardless of their possibility to be fundamental-dependent ones. It is obvious that all the objects indirectly grounded in the fundamental objects are at least relatively nonfundamental. (They also may be relatively fundamental but may not if there are infinite chains of groundings). The point is that these objects are viewed as non-fundamental. The knowledge of these objects is naturally to be called analogously.

Now we can define metaphysics as the fundamental knowledge in the above sense of the term. (The possibility of such knowledge is obvious for 
the author of this paper but is not discussed here. What is discussed here is not the possibility but the essence of metaphysics). Accordingly, the non-fundamental knowledge deserves the name of the non-metaphysical.

To demonstrate non-fundamentality of natural sciences it is sufficient to show nonfundamentality of physics. If physical knowledge, on the contrary, is fundamental then the primacy of fundamental physics as it is formulated by J. Ladyman and D. Ross [9, p.44] looks quite plausible. The subject matter of physics is the aggregate of physical objects. The notion of a physical object indicates anything existing, discernible and physical: may it be things, relations, processes, structures, laws of nature etc. Physical objects are discrete in the sense that they differ from all other objects, and can be distinguished from them. They can combine themselves, be transformed into others, or be separated from others but in the process, they retain a relative identity. There are no isolated physical objects, in other words, those that are not correlated with any other physical objects. The above is a weak formulation of the generally recognized in modern physics and philosophy of science principle of the causal closure of physical reality. According to this principle the cause of any physical object (in our sense of the term "object") may be exclusively physical. The weak formulation means that any physical object has at least some defining relations with other physical objects. It follows from this that the identifiers of each physical object are its qualitative and quantitative relations with other physical objects. These relations are concrete that is, they have the status of one of the plurality of formally possible states.

Any identifying physical relation is not unique (and therefore concrete) in two senses. First it is not the only relation of a physical object. Second it is not the relation of a unique object i.e. the relation of an object with itself. This is so just because physical objects are essentially different. Different objects cannot have one relation between them. The development of physics shows no decrease in the number of entities the existence of which is postulated. On the contrary, the theoretical field of physics becomes more and more complicated. It is unlikely the evidence in favour of the physics fundamentality.

The concreteness of identifying physical relations indicates that physical grounding take place. It is necessary for the physical object to be specified and the power of specification belongs to some other objects. And so all physical objects are grounded, that is, their existence is due to the existence of other objects. This entails that no physical objects are directly fundamental. Neither they are fundamentally-dependent. Because of the closure of physical reality only physical objects can provide the ground for a physical object. From the physical world as it is now understood therefore there is no access to the non-physical world (where one could discover fundamental objects). The general conclusion is that physical objects viewed as purely physical can be neither fundamental nor fundamentally-dependent. And so physics, like other natural sciences, deals with non-fundamental objects and is not a fundamental knowledge. In other words it is not and cannot be real metaphysics or include metaphysics as its fundamental part.

It is possible though that there is still something metaphysical within physics. One should try the opportunity to find metaphysics within physics not in the objects of physical relations but in the relations of physical objects - abstracted and mathematically expressed. It may be that math formulas used by physicists or their possible generalizations express ultimate reality. Is it right to look for the fundamental reality among mathematical structures?

Let's define mathematical objects as any entities (real or imaginable) distinguishable and representable by mathematicians. There are obvious elementary objects in mathematics (opposite to physics) and they are the sets. Are the sets fundamental in the above sense? It is necessary to find out what grounds the sets (provided there is such grounding). A set is commonly defined in mathematics (in the naive version of set theory) as distinct objects gathered together. This statement establishes two necessary and sufficient conditions for the existence of sets. First, a set is anything. Second, a set is a whole. In axiomatic theories of sets, these two conditions correspond to two basic building blocks of a theory - the set itself and the membership relation. The set itself is surely fundamental, it is just what exists and it needs nothing to ground it. What exists is not the set however but the sets. The presence of other sets is determined by the relation of membership. We can't go from one set to another without this relationship. The necessity of membership relations means that each set is grounded by some other sets, which, in turn, are grounded by 
their own collections of defining sets. Ultimately, each set is grounded by an infinite plurality of other sets. The sets by no means are identical with their grounds. Neither are they grounded by finite sequences of grounds. So all sets are not directly fundamental, they are not fundamentaldependent as well.

Now let's take into account that any mathematical object is a set. What have been said about sets is valid for all mathematical objects. It would be justified to say that mathematical objects are neither fundamental nor fundamental-dependent in the above sense. Therefore mathematics alongside with natural sciences is not a fundamental knowledge. It is not metaphysics; it cannot play this role by its nature. One could say that mathematics is not abstract enough; it has some special presuppositions which are not obvious. Hence it needs some fundamental grounding and such grounding may be only non-mathematical.

A similar conclusion looks plausible for natural sciences. The latter are not able to produce metaphysical data because they explore exclusively non-fundamental entities. If metaphysics is possible, if it is not a chimera it must deal with reality that lies, at least partly, outside the sphere of natural sciences. It is obvious in this context that natural sciences do not represent the full reality; they have limits caused by their subjectmatter. This leads to two principal statements on metaphysics. 1) Only the speculative metaphysics (if such metaphysics is possible) has the means to solve metaphysical problems. 2) Metaphysics (if it is a valid metaphysics) relies entirely on its own foundation.

This does not mean that natural sciences describe the world fallaciously. They describe the world from their own side. Attempts to cover the whole world from this side are probably inevitable, but they will only lead to an infinite expansion and deepening of knowledge. It is the efforts to reach the bottom which is not there. Note that the bottom does not exist for natural sciences and mathematics (and generally for all special theoretical disciplines). Whether it exists at all is the major question and the basis for justification of the speculative kind of metaphysics. Natural sciences represent the view from the side of empirical data, from "our (a viewer's) side" of the world extending deep into reality. Metaphysics is the view, one can say, from the back side of the world. Whether this side is accessible to human mind, whether it exists at all - the questions are debatable but not meaningless.

Metaphysics and natural sciences have the opposite points of departure but they must meet somewhere since they explore one and the same world. Though these two kinds of knowledge do not coincide, they have to be correlated. One may think about the necessary analogies between metaphysical and physical notions, structures, dependencies. This means that the representatives of metaphysics and natural sciences need to be aware of the results of the other area of knowledge, to take them into account, to coordinate their constructive work. Yet they have to proceed from different theoretical foundations and to posit different tasks.

\section{Conclusion}

Natural sciences are undoubtedly useful. They are powerful. They give a lot of benefits to mankind. In particular, they vastly expand our understanding of reality in which we find ourselves. But they are ineffective as the means of metaphysical knowledge. They will never give us a complete picture of the world. They are not metaphysics. Metaphysics (if it is realizable) must be something principally different from natural knowledge.

\section{References}

1. Andersen, F. \& Becker A. J. R. (2016). Metaphysics Within Science: Against Radical Naturalism. Metaphilosophy, 47(2), 159-180. doi: 10.1111/meta.12175

2. Bennett, K. (2016). There is no special problem with metaphysics. Philosophical Studies, 173(1), 2137. doi: $10.1007 / \mathrm{s} 11098-014-0439-0$

3. Benovsky, J. (2013). From Experience to Metaphysics: On Experience-based Intuitions and their Role in Metaphysics. Noûs, 49(3), 684-697. doi: 10.1111/nous.12024

4. Bradley, F. H. (1916). Appearance and Reality: A metaphysical Essay. London: Allen \& Unwin. 
5. Chakravartty, A. (2013). On the Prospects of Naturalized Metaphysics. In D. Ross, J. Ladyman \& H. Kincaid (Eds.), Scientific Metaphysics (pp. 27-50). Oxford: Oxford University Press.

6. Esfield, M. (2006). The Impact of Science on Metaphysics and its Limits. Abstracta, 2(2), 86-101.

7. Faye, J. (2012). The Role of Philosophy in a Naturalized World. European Journal of Analytic Philosophy, 8(1), 60-76.

8. Hawking, S. \& Mlodinow, L. (2010). The grand design. New York: Bantam Books.

9. Ladyman, J. \& Ross, D. (2007). Every Thing Must Go: Metaphysics Naturalized. Oxford: Oxford University Press.

10. Lowe, E. J. (2011). The rationality of metaphysics. Synthese, 178(1), 99-109. doi: 10.1007/s11229009-9514-Z

11. Morganti, M. (2015). Science-based Metaphysics: On Some Recent Anti-metaphysical Claims. Philosophia Scientiæ,19-1, 57-70. doi: 10.4000/philosophiascientiae.1038

12. Paul, L. A. (2012). Metaphysics as modelling: the handmaiden's tale. Philosophical Studies, 160(1), 1-29. doi: 10.1007/s11098-012-9906-7

13. Weinberg, S. (1992). Dreams of a final theory. New York: Pantheon Books.

14. Wilson, J. (2016). The Question of Metaphysics. The Philosophers' Magazine, 74, 90-96. doi: $10.5840 /$ tpm201674107

(C) A. Kulieshov

Received 2017-02-15, Accepted 2017-02-26, Published online 2017-02-28 